
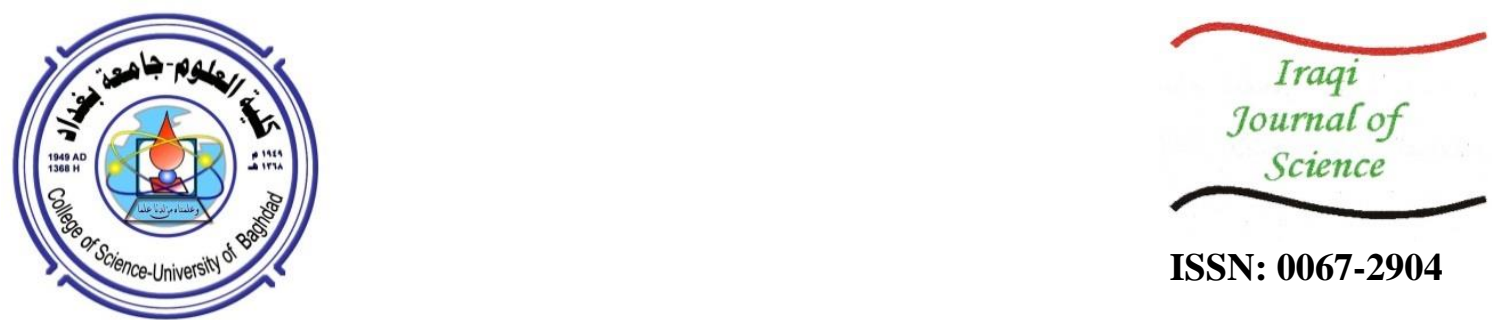

ISSN: 0067-2904

\title{
Solution of Time-Varying Index-2 Linear Differential Algebraic Control Systems Via A Variational Formulation Technique
}

\author{
Radhi A. Zaboon, Ghazwa F. Abd* \\ Department of Mathematics, College of Science, Mustansiriyah University, Baghdad, Iraq
}

Received: 26/1/2021

Accepted: 22/4/2021

\begin{abstract}
This paper deals with finding an approximate solution to the index-2 time-varying linear differential algebraic control system based on the theory of variational formulation. The solution of index-2 time-varying differential algebraic equations (DAEs) is the critical point of the equivalent variational formulation. In addition, the variational problem is transformed from the indirect into direct method by using a generalized Ritz bases approach. The approximate solution is found by solving an explicit linear algebraic equation, which makes the proposed technique reliable and efficient for many physical problems. From the numerical results, it can be implied that very good efficiency, accuracy, and simplicity of the present approach are obtained.
\end{abstract}

Keywords: Control problems, Direct method of calculus of variation, Generalized Ritz method, Index-two time-varying linear differential algebraic equations, Variational formulation.

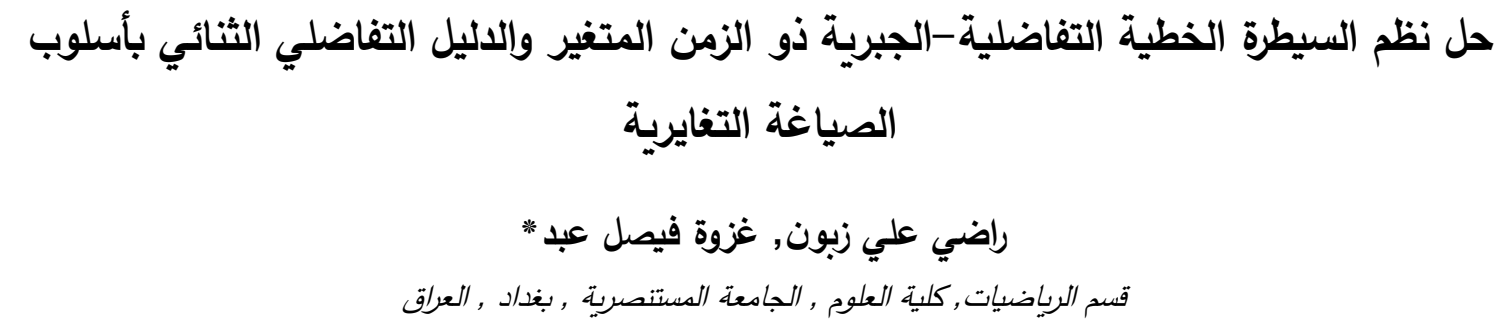

الخلاصة

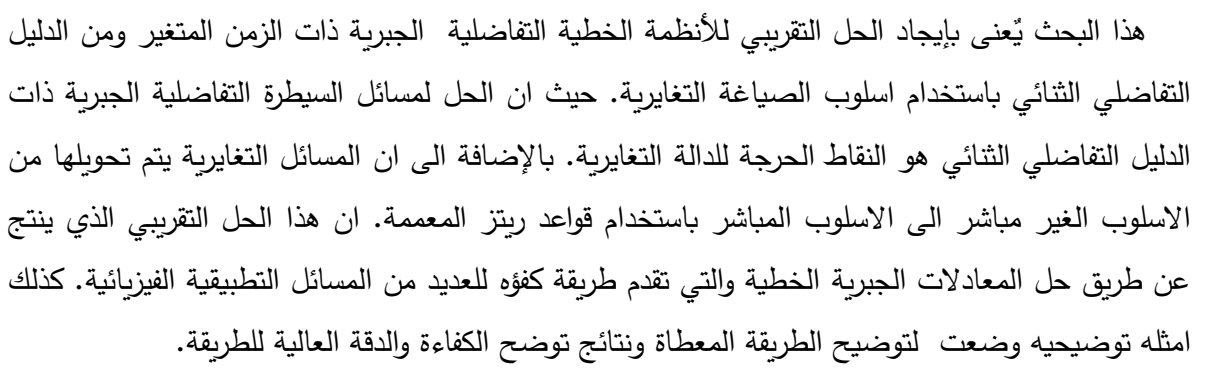

\section{Introduction}

Many real life problems can be modelled as a differential algebraic (control) system. Finding a novel reliable and efficient technique for solving differential algebraic equations 
has become an interesting aim for mathematicians and engineers. Numerical methods that solve higher index differential algebraic equations can be found in literature [1-7]. Many of these methods were based on the index reduction technique to avoid the difficulties in the higher index differential algebraic equations. Time- varying linear differential algebraic equations is a subject of many real life problems and has been the subject of many researchers in recent years $[8,9,10]$. An efficient and easily implemented technique to solve some classes of DAEs (index-1), approximately using non-classical variational formulation approach, was developed [11, 12,13]. The aim of this work is to extend and develop the results of the latter three studies to solve higher index time-varying linear differential algebraic control equations, especially for index-2 problems, without using the reducing technique which is not applicable for many real life problems. Since the proposed DAEs problem has the non-symmetrical time-derivative linear operator with respect to the classical bilinear form, a new bilinear form, based on the old one, is taken to ensure the necessary requirements for the existences of the variational problem corresponding to the given constrained problem.

\section{Basic Concepts}

Let $X$ and $Y$ be linear spaces and $: \mathbb{D}(L) \subset X \rightarrow \mathbb{R}(L)$ in $Y$, then $L$ is called symmetric with respect to the bilinear form $\langle a, b\rangle$ if

$$
\langle L a, b\rangle=\langle L b, a\rangle \text { satisfied for } a, b \in \mathbb{D}(L) .
$$

Moreover, a bilinear $\langle a, b\rangle$ is called non-degenerate on $X$ and $Y$ if the following two conditions are satisfied:

Firstly, for every $a \in X,\langle a, \bar{b}\rangle=0$, then $\bar{b}=0$, and secondly, for every $b \in Y,\langle\bar{a}, b\rangle=$ 0 , then $\bar{a}=0[11,14]$.

\section{Problem Formulation}

Consider the semi explicit linear descriptor system

$E \dot{x}=A x+B u+f(t)$,

where $E, A \in R^{m \times n}$, with $\operatorname{rank}(E)=n_{0}<n, x \in R^{n}, u \in R^{r}, B \in R^{m \times r}, f \in C\left(I ; R^{m}\right)$.

Since the rank $(E)=n_{0}$, it follows from [9], [15], and [16] that there exists unitary matrices $U \in R^{m \times m}$ and $V \in R^{n \times n}$ such that

$E=U\left(\begin{array}{ll}\Sigma & 0 \\ 0 & 0\end{array}\right) V^{T}, \Sigma=\operatorname{diag}\left(\delta_{1}, \delta_{2}, \ldots, \delta_{n_{0}}\right)$ and $\delta_{1} \geq \delta_{2} \geq . . \geq \delta_{n_{0}}>0$,

With the setting $\mathbb{P}=V, \mathbb{Q}=U^{-1}$, where $\mathbb{Q} E \mathbb{P}=\left(\begin{array}{ll}\Sigma & 0 \\ 0 & 0\end{array}\right)$, one gets

$\mathbb{P}^{-1} x=\left(\begin{array}{l}x_{1} \\ x_{2}\end{array}\right), x_{1} \in R^{n_{0}}, x_{2} \in R^{n-n_{0}}$, hence

$$
\mathbb{Q} E \mathbb{P}\left(\begin{array}{l}
\dot{x}_{1} \\
\dot{x}_{2}
\end{array}\right)=\mathbb{Q} A \mathbb{P}\left(\begin{array}{l}
x_{1} \\
x_{2}
\end{array}\right)+\mathbb{Q} B u+\mathbb{Q} f(t)
$$

Set $\mathbb{Q} A \mathbb{P}=\left(\begin{array}{ll}\tilde{A}_{11} & \tilde{A}_{12} \\ \tilde{A}_{21} & \tilde{A}_{22}\end{array}\right), \mathbb{Q} B=\left(\begin{array}{c}\tilde{B}_{1} \\ \tilde{B}_{2}\end{array}\right), \mathbb{Q} f=\left(\begin{array}{c}\tilde{f}_{1}(t) \\ \tilde{f}_{2}(t)\end{array}\right)$,

$$
\left(\begin{array}{ll}
\Sigma & 0 \\
0 & 0
\end{array}\right)\left(\begin{array}{l}
\dot{x}_{1} \\
\dot{x}_{2}
\end{array}\right)=\left(\begin{array}{ll}
\tilde{A}_{11} & \tilde{A}_{12} \\
\tilde{A}_{21} & \tilde{A}_{22}
\end{array}\right)\left(\begin{array}{l}
x_{1} \\
x_{2}
\end{array}\right)+\left(\begin{array}{c}
\tilde{B}_{1} \\
\tilde{B}_{2}
\end{array}\right) u+\left(\begin{array}{c}
\tilde{f}_{1}(t) \\
\tilde{f}_{2}(t)
\end{array}\right)
$$

Hence, the semi explicit system is transformed into differential algebraic systems:

$$
\begin{aligned}
& \dot{x}_{1}=A_{11} x_{1}+A_{12} x_{2}+B_{1} u+f_{1} \\
& 0=A_{21} x_{1}+A_{22} x_{2}+B_{2} u+f_{2}
\end{aligned}
$$

where $A_{11}=\Sigma^{-1} \tilde{A}_{11}, \quad A_{12}=\Sigma^{-1} \tilde{A}_{12}, \quad A_{21}=\tilde{A}_{21}, \quad A_{22}=\tilde{A}_{22}$,

$$
f_{1}=\Sigma^{-1} \tilde{f}_{1}, \quad f_{2}=\tilde{f}_{2}, \quad B_{1}=\Sigma^{-1} \widetilde{B}_{1}, \quad B_{2}=\tilde{B}_{2} .
$$

If there exists $A_{22}=0$ is invertible matrix with $\left(A_{21} A_{12}\right)$ or $A_{22}$ is not invertible matrix with $\left(A_{21} A_{12}\right)^{-1}$, then the system (1) and (2) are index two linear DAEs with control $u$. 
From the Jacobean of the algebraic constraint with respect to $x_{2}$, one can use the implicit function theorem $[5,17,18]$ to solve the following:

$$
x_{2}=\mathcal{L}\left(t, x_{1}, u, \dot{u}\right) \triangleq-\left(A_{21} A_{12}\right)^{-1}\left[A_{21} A_{11} x_{1}+A_{21} B_{1} u+A_{21} f_{1}+B_{2} \dot{u}+\dot{f}_{2}\right]
$$

Thus, from (1), (2), and (3), one have

$$
\begin{gathered}
\dot{x}_{1}=A_{11} x_{1}+A_{12} \mathcal{L}(t, x, u, \dot{u})+B_{1} u+f_{1} \\
0=A_{21} x_{1}+A_{22} x_{2}+B_{2} u+f_{2}
\end{gathered}
$$

where the class of consistent initial condition at $t=t_{0}$ is defined according to the given algebraic constraint (2), as follows:

$$
\begin{gathered}
\omega^{0}=\left\{\left(x_{1}^{0}, x_{2}^{0}\right) \in R^{\operatorname{rank}(E)} \times R^{n-\operatorname{rank}(E)} \mid A_{21} x_{1}\left(t_{0}\right)+B_{2} u\left(t_{0}\right)+f_{2}\left(t_{0}\right)=0,\right. \\
\left.x_{2}\left(t_{0}\right)=\mathcal{L}\left(t_{0}, x_{1}\left(t_{0}\right), u\left(t_{0}\right), \dot{u}\left(t_{0}\right)\right)\right\}
\end{gathered}
$$

Note that, if there is an interest in finding the explicit expression for $\dot{x}_{2}$ to obtain the statespace $\left(\dot{x}_{1}, \dot{x}_{2}\right)$, then one has to derive (3) with respect to t, as follows:

$\dot{x}_{2}=\frac{d}{d t} \mathcal{L}\left(t, x_{1}, u, \dot{u}\right)=\frac{\partial \mathcal{L}}{\partial t}+\frac{\partial \mathcal{L}}{\partial x_{1}} \dot{x}_{1}+\frac{\partial \mathcal{L}}{\partial u} \dot{u}+\frac{\partial \mathcal{L}}{\partial \dot{u}} \ddot{u}$

For $u \in C^{2}\left[I, R^{r}\right], t \in I=\left[t_{0}, t_{1}\right], f \in\left[I, R^{n-n_{0}}\right]$

$$
\begin{aligned}
\dot{x}_{2}=\frac{\partial \mathcal{L}}{\partial t} & +\frac{\partial \mathcal{L}}{\partial x_{1}}\left[A_{11} x_{1}+A_{12} x_{2}+B_{1} u+f_{1}\right]+\frac{\partial \mathcal{L}}{\partial u} \dot{u}+\frac{\partial \mathcal{L}}{\partial \dot{u}} \ddot{u} \\
= & \left(\frac{\partial \mathcal{L}}{\partial x_{1}} A_{11}\right) x_{1}+\left(\frac{\partial \mathcal{L}}{\partial x_{1}} A_{12}\right) x_{2}+\left(\frac{\partial \mathcal{L}}{\partial x_{1}} B_{1}\right) u+\frac{\partial \mathcal{L}}{\partial x_{1}} f_{1}+\frac{\partial \mathcal{L}}{\partial u} \dot{u}+\frac{\partial \mathcal{L}}{\partial \dot{u}} \ddot{u}+\frac{\partial \mathcal{L}}{\partial t}
\end{aligned}
$$

Then systems (1) and (2) are equivalent to state- space differential equation defined on manifold:

$$
\begin{gathered}
\left(\begin{array}{l}
\dot{x}_{1} \\
\dot{x}_{2}
\end{array}\right)=\left(\begin{array}{cc}
A_{11} & A_{12} \\
\frac{\partial \mathcal{L}}{\partial x_{1}} A_{11} & \frac{\partial \mathcal{L}}{\partial x_{1}} A_{12}
\end{array}\right)\left(\begin{array}{l}
x_{1} \\
x_{2}
\end{array}\right)+\left(\begin{array}{c}
B_{1} \\
\frac{\partial \mathcal{L}}{\partial x_{1}} B_{1}
\end{array}\right) u+\left(\begin{array}{c}
0 \\
\frac{\partial \mathcal{L}}{\partial u}
\end{array}\right) \dot{u} \\
+\left(\begin{array}{c}
0 \\
\frac{\partial \mathcal{L}}{\partial \dot{u}}
\end{array}\right) \ddot{u}+\left(\begin{array}{c}
f_{1} \\
\frac{\partial \mathcal{L}}{\partial x_{1}} f_{1}+\frac{\partial \mathcal{L}}{\partial t}
\end{array}\right)
\end{gathered}
$$

where $x_{2}(t)=\mathcal{L}\left(t, x_{1}, u, \dot{u}\right)$, subject to the manifold

$$
A_{21} x_{1}(t)+B_{2} u(t)+f_{2}(t)=0
$$

As one can see, the terms $\dot{u}, \ddot{u}$ are not appropriate for an application point of view, and the usage of the implicit function theorem to reduce the number of variables and estimate $x_{2}$ by (3) is better than solving problems (5) and (6).

\section{Index-2 Time-Varying DAEs and their Variational Formulations}

The main theme of this section is to discuss the solvability of index-2 time-varying DAEs using the variational formulation approach.

We are looking for a suitable function, such that its critical points lead to a solution to the proposed problem and vice-versa.

Define

$$
\begin{aligned}
& \dot{x}_{1}(t)=A_{11}(t) x_{1}(t)+A_{12}(t) x_{2}(t)+B_{1}(t) u(t)+f_{1}(t) \\
& 0=A_{21}(t) x_{1}(t)+B_{2}(t) u(t)+f_{2}(t)
\end{aligned}
$$

where $A_{21}(t) A_{12}(t)$ is a non - singular matrix $\forall t \in I$, $B_{2}(t) u(t)+f_{2}(t) \in \operatorname{Range}\left(A_{21}(t)\right) u(t) \in \Delta_{u}$, where $\Delta_{u}$ is the class of admissible control defined according to the given problem.

If $u(t) \in c^{\prime}\left(I, R^{r}\right), f_{2} \in c\left(I, R^{n}\right), f_{2} \in c^{\prime}\left(I, R^{n-n_{0}}\right)$, and $I=\left[t_{0}, t_{f}\right], t_{f}>t_{0}$, one can have the following

$$
\begin{aligned}
& 0=\dot{A}_{21} x_{1}+A_{21} \dot{x}_{1}+\dot{B}_{2} u+B_{2} \dot{u}+\dot{f}_{2} \\
& =\dot{A}_{21} x_{1}+A_{21}\left(A_{11} x_{1}+A_{12} x_{2}+B_{1} u+f_{1}\right)+\dot{B}_{2} u+B_{2} \dot{u}+\dot{f}_{2}
\end{aligned}
$$




$$
=\dot{A}_{21} x_{1}+A_{21} A_{11} x_{1}+A_{21} A_{12} x_{2}+A_{21} B_{1} u+A_{21} f_{1}+\dot{B}_{2} u+B_{2} \dot{u}+\dot{f}_{2}
$$

Since the problem is index 2, i.e. $\left(A_{21} A_{12}\right)$ is invertible matrix, then (9) is solvable and gives that

$$
\begin{array}{r}
x_{2}(t)=-\tilde{A}(t) x_{1}(t)-\tilde{B}(t) u(t)-\left(\left(A_{21}(t) A_{12}(t)\right)^{-1} A_{21}(t)\right) f_{1}(t) \\
-\left(\left(A_{21}(t) A_{12}(t)\right)^{-1} B_{2}(t)\right) \dot{u}(t)-\left(A_{21}(t) A_{12}(t)\right)^{-1} \dot{f}_{2}(t)
\end{array}
$$

where $\tilde{A}=\left(A_{21}(t) A_{12}(t)\right)^{-1}\left(A_{21}(t) A_{11}(t)+\dot{A}_{21}(t)\right)$,

$$
\tilde{B}=\left(A_{21}(t) A_{12}(t)\right)^{-1}\left(A_{21}(t) B_{1}(t)+\dot{B}_{2}(t)\right) \text {. }
$$

This leads to $x_{2}(t)=\mathcal{L}\left(x_{1}, u, \dot{u}\right), u(t) \in \Delta_{u}, f \in c^{\prime}\left(I, R^{n-n_{0}}\right)$, $I=\left[t_{0}, t_{f}\right], t_{0}, t_{f}$ are given and real numbers with $t_{0}<t_{f}$.

The selection of a consistent initial condition is based on the nature of equations (8), as:

$0=A_{21}\left(t_{0}\right) x_{1}\left(t_{0}\right)+B_{2}\left(t_{0}\right) u\left(t_{0}\right)+f_{2}\left(t_{0}\right)$

$x_{2}\left(t_{0}\right)=\mathcal{L}\left(x_{1}\left(t_{0}\right), u\left(t_{0}\right), \dot{u}\left(t_{0}\right)\right)$, for a given $x_{1}\left(t_{0}\right), u\left(t_{0}\right), \dot{u}\left(t_{0}\right)$

Next, we define

$$
\begin{aligned}
\omega^{0}= & \left\{\left(x_{1}\left(t_{0}\right), x_{2}\left(t_{0}\right)\right) \in R^{\operatorname{rank}(E)} \times R^{n-\operatorname{rank}(E)} \mid x_{2}\left(t_{0}\right)=\mathcal{L}\left(x_{1}(0), u(0), \dot{u}(0)\right)\right. \\
= & -\tilde{A}\left(t_{0}\right) x_{1}\left(t_{0}\right)-\tilde{B}\left(t_{0}\right) u\left(t_{0}\right)-\left(\left(A_{21}\left(t_{0}\right) A_{12}\left(t_{0}\right)\right)^{-1} A_{21}\left(t_{0}\right)\right) f_{1}\left(t_{0}\right) \\
& -\left(\left(A_{21}\left(t_{0}\right) A_{12}\left(t_{0}\right)\right)^{-1} B_{2}\left(t_{0}\right)\right) \dot{u}\left(t_{0}\right)-\left(A_{21}\left(t_{0}\right) A_{12}\left(t_{0}\right)\right)^{-1} \dot{f}_{2}\left(t_{0}\right), \\
& \text { for a given } \left.f_{1}\left(t_{0}\right), f_{2}\left(t_{0}\right), u\left(t_{0}\right), \dot{u}\left(t_{0}\right)\right\} .
\end{aligned}
$$

We redefine the constrained DAEs as

$$
\begin{aligned}
& L_{1} x_{1}(t)=G_{1}(t), \\
& L_{2} x_{1}(t)=G_{2}(t), \\
& L_{3} x_{1}\left(t_{0}\right)=G_{3}\left(t_{0}\right)
\end{aligned}
$$

where

$$
\begin{aligned}
& L_{1} x_{1}(t) \triangleq \frac{d}{d t} x_{1}(t)+\left(A_{12}(t) \tilde{A}(t)-A_{11}(t)\right) x_{1}(t) \\
& L_{2} x_{1}(t) \triangleq-A_{21}(t) x_{1}(t) \\
& L_{3} x_{1}\left(t_{0}\right) \triangleq L_{2} x_{1}\left(t_{0}\right) \\
& G_{1}(t) \triangleq B_{1}(t) u(t)+f_{1}(t)-\left(\left(A_{21}(t) A_{12}(t)\right)^{-1} A_{21}(t)\right) f_{1}(t) \\
& \quad-\left(\left(A_{21}(t) A_{12}(t)\right)^{-1} B_{2}(t)\right) \dot{u}(t)-\left(A_{21}(t) A_{12}(t)\right)^{-1} \dot{f}_{2}(t) \\
& G_{2}(t) \triangleq B_{2}(t) u(t)+f_{2}(t) \quad, G_{3}\left(t_{0}\right)=B_{3}\left(t_{0}\right) u\left(t_{0}\right)+f_{3}\left(t_{0}\right) \\
& \text { Set } L=\left(L_{1}, L_{2}, L_{3}\right)^{T} \\
& L: D(L) \subset C\left(I, R^{n_{0}}\right) \rightarrow \operatorname{Rang}(L) \\
& D(L)=\left\{x_{1} \in C^{\prime}\left(I, R^{n_{0}}\right) \mid x_{1}\left(t_{0}\right) \in \omega^{0}\right\} \subset C\left(I, R^{n_{0}}\right) \forall u(t) \in c^{\prime}\left(I, R^{r}\right), f_{2} \in c\left(I, R^{n}\right), \\
& \quad f_{2} \in c^{\prime}\left(I, R^{n-n_{0}}\right) .
\end{aligned}
$$

Since the operator $\frac{d}{d t}$ is appeared in $L_{1} x_{1}(t)$, the linear operator $L$ is not symmetric with the given usual bilinear form basic concept. Hence, no variational formulation exists unless one can redefine the linear operator or its bilinear form [11, 19].

To create a functional (variational) equivalent to a linear problem $L u=f$, where $L$ is not symmetric with respect to the chosen bilinear form, by the functional $F\left(x_{1}, \mathcal{L}, u, \dot{u}, f, \dot{f}\right)=$ $F\left[x_{1}\right]$, we have:

$$
\begin{aligned}
& \left.F\left[x_{1}\right]=\int_{t_{0}}^{t_{f}}\left[\begin{array}{lll}
\frac{1}{2}\left(L_{1}^{T} x_{1}(t)\right. & L_{2}^{T} x_{1}(t) & L_{3}^{T} x_{1}\left(t_{0}\right)
\end{array}\right)\left(\begin{array}{c}
L_{1} x_{1}(t) \\
L_{2} x_{1}(t) \\
L_{3} x_{1}\left(t_{0}\right)
\end{array}\right)-\left(\begin{array}{lll}
G_{1}^{T}(t) & G_{2}^{T}(t) & G_{3}^{T}\left(t_{0}\right)
\end{array}\right)\left(\begin{array}{c}
L_{1} x_{1}(t) \\
L_{2} x_{1}(t) \\
L_{3} x_{1}\left(t_{0}\right)
\end{array}\right)\right] d t \\
& F\left[x_{1}\right]=\int_{t_{0}}^{t_{f}}\left[\frac{1}{2} L_{1}^{T} x_{1}(t) L_{1} x_{1}(t)+\frac{1}{2} L_{2}^{T} x_{1}(t) L_{2} x_{1}(t)+\frac{1}{2} L_{3}^{T} x_{1}\left(t_{0}\right) L_{3} x_{1}\left(t_{0}\right)-G_{1}^{T}(t) L_{1} x_{1}(t)-\right. \\
& \left.G_{2}^{T}(t) L_{2} x_{1}(t)-G_{3}^{T}\left(t_{0}\right) L_{3} x_{1}\left(t_{0}\right)\right] d t
\end{aligned}
$$




$$
\begin{aligned}
=\frac{1}{2}\left\langle L_{1} x_{1}(t),\right. & \left.L_{1} x_{1}(t)\right\rangle+\frac{1}{2}\left\langle L_{2} x_{1}(t), L_{2} x_{1}(t)\right\rangle+\frac{1}{2}\left\langle L_{3} x_{1}\left(t_{0}\right), L_{3} x_{1}\left(t_{0}\right)\right\rangle-\left\langle G_{1}(t), L_{1} x_{1}(t)\right\rangle \\
- & \left\langle G_{2}(t), L_{2} x_{1}(t)\right\rangle-\left\langle G_{3}\left(t_{0}\right), L_{3} x_{1}\left(t_{0}\right)\right\rangle .
\end{aligned}
$$

where $\langle x, y\rangle=\int_{t_{0}}^{t_{f}} x^{T} y d t, x(t), y(t) \in C\left[t_{0}, t_{f}\right]$.

We define the first variation, due to the linear part of the increment of the functional $F\left[x_{1}(t)\right]$, as:

$$
\begin{aligned}
\delta F\left(x_{1}(t)\right)= & F\left[x_{1}+\delta x_{1}\right]-\left.F\left[x_{1}\right]\right|_{\text {linear part in } \delta x_{1}}=0 \\
\delta F=\frac{1}{2}\left\langleL _ { 1 } \left( x_{1}\right.\right. & \left.\left.+\delta x_{1}\right), L_{1}\left(x_{1}+\delta x_{1}\right)\right\rangle+\frac{1}{2}\left\langle L_{2}\left(x_{1}+\delta x_{1}\right), L_{2}\left(x_{1}+\delta x_{1}\right)\right\rangle \\
& +\frac{1}{2}\left\langle L_{3}\left(x_{1}\left(t_{0}\right)+\delta x_{1}\left(t_{0}\right)\right), L_{3}\left(x_{1}\left(t_{0}\right)+\delta x_{1}\left(t_{0}\right)\right)\right\rangle-\left\langle G_{1}(t), L_{1}\left(x_{1}+\delta x_{1}\right)\right\rangle \\
& -\left\langle G_{2}(t), L_{2}\left(x_{1}+\delta x_{1}\right)\right\rangle-\left\langle G_{3}\left(t_{0}\right), L_{3}\left(x_{1}\left(t_{0}\right)+\delta x_{1}\left(t_{0}\right)\right)\right\rangle \\
& -\left[\frac{1}{2}\left\langle L_{1} x_{1}(t), L_{1} x_{1}(t)\right\rangle+\frac{1}{2}\left\langle L_{2} x_{1}(t), L_{2} x_{1}(t)\right\rangle+\frac{1}{2}\left\langle L_{3} x_{1}\left(t_{0}\right), L_{3} x_{1}\left(t_{0}\right)\right\rangle\right. \\
& \left.-\left\langle G_{1}(t), L_{1} x_{1}(t)\right\rangle-\left\langle G_{2}(t), L_{2} x_{1}(t)\right\rangle-\left\langle G_{3}\left(t_{0}\right), L_{3} x_{1}\left(t_{0}\right)\right\rangle\right] .
\end{aligned}
$$

Such that

$$
\begin{aligned}
\left\langle L_{1}\left(x_{1}+\delta x_{1}\right),\right. & \left.L_{1}\left(x_{1}+\delta x_{1}\right)\right\rangle \\
& =\left\langle L_{1} x_{1}, L_{1} x_{1}\right\rangle+\left\langle L_{1} x_{1}, L_{1} \delta x_{1}\right\rangle+\left\langle L_{1} \delta x_{1}, L_{1} \delta x_{1}\right\rangle+\left\langle L_{1} \delta x_{1}, L_{1} x_{1}\right\rangle
\end{aligned}
$$

Since $\left\langle L_{1} \delta x_{1}, L_{1} \delta x_{1}\right\rangle$ is non linear in the term of $\delta x_{1}$, then

And by the same way

$$
\left\langle L_{1}\left(x_{1}+\delta x_{1}\right), L_{1}\left(x_{1}+\delta x_{1}\right)\right\rangle=\left\langle L_{1} x_{1}, L_{1} x_{1}\right\rangle+2\left\langle L_{1} x_{1}, L_{1} \delta x_{1}\right\rangle .
$$

$$
\begin{aligned}
& \left\langle L_{2}\left(x_{1}+\delta x_{1}\right), L_{2}\left(x_{1}+\delta x_{1}\right)\right\rangle=\left\langle L_{2} x_{1}, L_{2} x_{1}\right\rangle+2\left\langle L_{2} x_{1}, L_{2} \delta x_{1}\right\rangle \\
& \left\langle L_{3}\left(x_{1}\left(t_{0}\right)+\delta x_{1}\left(t_{0}\right)\right), L_{3}\left(x_{1}\left(t_{0}\right)+\delta x_{1}\left(t_{0}\right)\right)\right\rangle \\
& \quad=\left\langle L_{3} x_{1}\left(t_{0}\right), L_{3} x_{1}\left(t_{0}\right)\right\rangle+2\left\langle L_{3} x_{1}\left(t_{0}\right), L_{3} \delta x_{1}\left(t_{0}\right)\right\rangle .
\end{aligned}
$$

where $x_{1}\left(t_{0}\right)$ is an arbitrary selection from the class of consistency initial condition $\omega^{0}$. Otherwise, one can assume it as fixed numbered and set $\delta x_{1}\left(t_{0}\right)=0$,

$$
\begin{aligned}
& \left\langle G_{1}(t), L_{1}\left(x_{1}+\delta x_{1}\right)\right\rangle=\left\langle G_{1}(t), L_{1} x_{1}(t)\right\rangle+\left\langle G_{1}(t), L_{1} \delta x_{1}(t)\right\rangle \\
& \left\langle G_{2}(t), L_{2}\left(x_{1}+\delta x_{1}\right)\right\rangle=\left\langle G_{2}(t), L_{2} x_{1}(t)\right\rangle+\left\langle G_{2}(t), L_{2} \delta x_{1}(t)\right\rangle \\
& \left\langle G_{3}\left(t_{0}\right), L_{3}\left(x_{1}\left(t_{0}\right)+\delta x_{1}\left(t_{0}\right)\right)\right\rangle=\left\langle G_{3}\left(t_{0}\right), L_{3} x_{1}\left(t_{0}\right)\right\rangle+\left\langle G_{3}\left(t_{0}\right), L_{3} \delta x_{1}\left(t_{0}\right)\right\rangle .
\end{aligned}
$$

From the above discussion, let us define $\delta F\left[x_{1}(t)\right]$ as:

$$
\begin{aligned}
& \delta F\left[x_{1}(t)\right]=\left\langle L_{1} x_{1}, L_{1} \delta x_{1}\right\rangle+\left\langle L_{2} x_{1}, L_{2} \delta x_{1}\right\rangle+\left\langle L_{3} x_{1}\left(t_{0}\right), L_{3} \delta x_{1}\left(t_{0}\right)\right\rangle-\left\langle G_{1}(t), L_{1} \delta x_{1}(t)\right\rangle \\
& \quad-\left\langle G_{2}(t), L_{2} \delta x_{1}(t)\right\rangle-\left\langle G_{3}\left(t_{0}\right), L_{3} \delta x_{1}\left(t_{0}\right)\right\rangle \\
& =\left\langle L_{1} x_{1}-G_{1}(t), L_{1} \delta x_{1}\right\rangle+\left\langle L_{2} x_{1}-G_{2}(t), L_{2} \delta x_{1}\right\rangle \\
& \quad+\left\langle L_{3} x_{1}\left(t_{0}\right)-G_{3}\left(t_{0}\right), L_{3} \delta x_{1}\left(t_{0}\right)\right\rangle \\
& \begin{array}{c}
\delta F\left[x_{1}(t)\right]=\int_{t_{0}}^{t_{f}}\left[\left(L_{1} x_{1}-G_{1}(t)\right)^{T} L_{1} \delta x_{1}+\left(L_{2} x_{1}-G_{2}(t)\right)^{T} L_{2} \delta x_{1}+\left(L_{3} x_{1}\left(t_{0}\right)-\right.\right. \\
\left.\left.G_{3}\left(t_{0}\right)\right)^{T} L_{3} \delta x_{1}\left(t_{0}\right)\right] d t
\end{array} \\
& =\int_{t_{0}}^{t_{f}}\left[\left(L_{1} x_{1}-G_{1}(t)\right)^{T}\left(L_{2} x_{1}-G_{2}(t)\right)^{T}\left(L_{3} x_{1}\left(t_{0}\right)-G_{3}\left(t_{0}\right)\right)^{T}\right]\left[\begin{array}{c}
L_{1} \delta x_{1} \\
L_{2} \delta x_{1} \\
L_{3} \delta x_{1}\left(t_{0}\right)
\end{array}\right] d t
\end{aligned}
$$

It is noticed that, if there is $x_{1} \in c^{\prime}\left(I, R^{n_{0}}\right)$ satisfying the operator equations (10)-(12), uniquely over the class $\omega^{0}$, then these equations will be identically satisfied.

Since the aim is to fix $x_{1} \in c^{\prime}\left(I, R^{n_{0}}\right)$, then this variational problem is well defined.

Also, since from the linearity in $x_{1}$,

$$
\Rightarrow L_{1} \delta x_{1}=\delta L_{1} x_{1}, \quad L_{2} \delta x_{1}=L_{2} \delta x_{1}, \quad \delta L_{3} x_{1}\left(t_{0}\right)=\delta L_{3} x_{1}\left(t_{0}\right)
$$

for arbitrary $\delta L_{1} x_{1}, \delta L_{2} x_{1}, \delta L_{3} x_{1}\left(t_{0}\right)$,

then 
$\delta F\left[x_{1}(t)\right]$

$=\left\langle\left[\begin{array}{llll}\left(L_{1} x_{1}-G_{1}(t)\right) & \left(L_{2} x_{1}-G_{2}(t)\right) & \left(L_{3} x_{1}\left(t_{0}\right)-G_{3}\left(t_{0}\right)\right)\end{array}\right],\left[\begin{array}{lll}L_{1} \delta x_{1} & L_{2} \delta x_{1} & L_{3} \delta x_{1}\left(t_{0}\right)\end{array}\right]\right\rangle$

For the arbitrary $\delta x_{1}$, the non-degeneracy property on the range and domain of the bilinear form, and the linearity property, we get

$\delta F\left[x_{1}(t)\right]=0$

$\Rightarrow\left[\begin{array}{llll}\left(L_{1} x_{1}-G_{1}(t)\right) & \left(L_{2} x_{1}-G_{2}(t)\right) & \left(L_{3} x_{1}\left(t_{0}\right)-G_{3}\left(t_{0}\right)\right)\end{array}\right]=\left[\begin{array}{lll}0 & 0 & 0\end{array}\right] \forall \delta x_{1}$

$\Rightarrow L_{1} x_{1}=G_{1}(t), \quad L_{2} x_{1}=G_{2}(t), \quad L_{3} x_{1}\left(t_{0}\right)=G_{3}\left(t_{0}\right), \quad x_{2}=L x_{1}$

$\Rightarrow L x_{1}-G(t)=0 \Leftrightarrow L x_{1}=G(t), L=\left(L_{1}, L_{2}, L_{3}\right)^{T}, G(t)=\left(G_{1}, G_{2}, G_{3}\right)$

It should be noticed that

1. If $x_{2}=L x_{1} \Rightarrow \delta x_{1}=\frac{\partial L}{\partial x_{1}} \delta x_{1}$ and this term should be inserted in the variational formulation.

2. If $x_{1}\left(t_{0}\right) \in \mathcal{M}\left(x_{1}\left(t_{0}\right)\right)$ (linear manifold of consistent initial conditions),

$\Rightarrow \delta x_{1}\left(t_{0}\right)=\left.\frac{\partial \mathcal{M}}{\partial x_{1}}\right|_{t=t_{0}} . \delta x_{1}\left(t_{0}\right)$,

3. $x_{1}\left(t_{0}\right)$ may also be assumed as fixed to produce that $\delta x_{1}\left(t_{0}\right)=0$ and this will not affect the previous results.

Then, if $x_{1}=G(t)$, with $L=\left(L_{1}, L_{2}, L_{3}\right)^{T}$ is the solution of the proposed problem, then $\left\langle 0, L_{1} \delta x_{1}\right\rangle=0 \Leftrightarrow \delta F\left[x_{1}(t)\right]=0$.

The other direction is clearly understood and the solution $x_{1} \in c^{\prime}\left(I, R^{n_{0}}\right)$ is a critical point of variable formulation (10).

From practical point of view, one has to evaluate the functional $F\left[x_{1}(t)\right]$ in order to find its critical points.

Moreover, critical points of a functional are equivalent to solve the necessary Euler equation corresponding to the given problem, which is difficult too. Thus, a direct method of variational problem is adapted to approximate the solution by a finite number of bases functions of separable Banach space $c(I, R)$, as:

$$
\begin{aligned}
& x_{1}^{j}=\sum_{i=0}^{m_{j}} a_{i}^{j} H_{i}^{j}(t), j=1,2, \ldots, n_{0}, m_{j} \text { arbitrary } \\
& x_{2}^{l}=\mathcal{L}\left(x_{1}^{j}, u, \dot{u}\right), l=1,2, \ldots, n-n_{0}, j=1,2, \ldots, n_{0}
\end{aligned}
$$

where $H_{i}^{j}$ is linearly independent bases function of time t.

By substituting (14) and (15) in (13), we have

$$
\begin{aligned}
F\left[x_{1}\right]= & F\left(x_{1}, \mathcal{L}, u, \dot{u}, f, \dot{f}\right) \\
& =F\left(a_{0}^{1}, a_{1}^{1}, a_{2}^{1}, \ldots, a_{m_{1}}^{1}, a_{0}^{2}, a_{1}^{2}, a_{2}^{2}, \ldots, a_{m_{2}}^{2}, \ldots, a_{0}^{n_{0}}, a_{1}^{n_{0}}, a_{2}^{n_{0}}, \ldots a_{m_{n_{0}}}^{n_{0}}\right)
\end{aligned}
$$

where $n=n_{0}+n-n_{0}$ is the total number of unknown variables.

The critical point of variational formulation (13) is then equivalent to find the derivative of the functional (16) with respect to $a_{i}^{j}, i=0, \ldots, m_{j}, j=1, \ldots, n_{0}$.

$$
\text { i.e. } \frac{\partial F}{\partial a_{i}^{j}}=0, \forall i=0, \ldots, m_{j}, j=1, \ldots, n_{0}
$$

Since the varitional formulation is of quadratic type, the linear system of algebraic equation was obtained from equation (14), with the class of consistency initial condition where the given functions $u(t), \dot{u}(t), f(t), \dot{f}(t)$ are selected from the class of admissible functions.

Once this system (17) is being solved for $a_{i}^{j}$, approximate solutions $x_{1}(t)$ and $x_{2}(t)$ are obtained according to equations (14) and (15) and hence the original solution of (7) and (8) is obtained approximately.

\section{Illustrations}

Example 5.1: (index 2 linear time-varying DAEs with given $u(t)$ over admissible class) Consider the linear time invariant index-2 semi explicit DAE problem [1], 


$$
\begin{aligned}
\left(\begin{array}{l}
\dot{x}_{11} \\
\dot{x}_{12}
\end{array}\right) & =\left(\begin{array}{cc}
-1 & 1 \\
0 & 0
\end{array}\right)\left(\begin{array}{l}
x_{11} \\
x_{12}
\end{array}\right)+\left(\begin{array}{c}
0 \\
1+2 t
\end{array}\right) x_{21}+\left(\begin{array}{l}
1 \\
0
\end{array}\right) u(t) \\
0 & =\left(\begin{array}{ll}
1 & 1
\end{array}\right)\left(\begin{array}{l}
x_{11} \\
x_{12}
\end{array}\right)-\left(e^{-t}\right)+u(t), t \in[0,1]
\end{aligned}
$$

Note that the variable $x_{21}$ does not appear in the algebraic constraint explicitly.

Then, by deriving the algebraic constraint with respect to $t$ and $u(t)=-$ sint we have that

$$
\begin{aligned}
& \frac{d}{d t}\left(0=x_{11}+x_{12}-e^{-t}-\sin t\right) \\
& \quad \Rightarrow 0=\dot{x}_{11}+\dot{x}_{12}+e^{-t}-\cos t
\end{aligned}
$$

We substitute (18) in (20) to get

$$
\begin{aligned}
& {\left[-x_{11}+x_{12}-\sin t\right]+[1+2 t] x_{21}+e^{-t}-\cos t=0} \\
& \Rightarrow x_{21}=\frac{x_{11}-x_{12}+\sin t-e^{-t}+\cos t}{1+2 t}=\mathcal{L}\left(x_{11}, x_{12}, t\right), \forall t \in[0,1] .
\end{aligned}
$$

The class of consistency initial condition is

$$
\begin{aligned}
& \omega^{0}=\left\{\left(x_{11}\left(t_{0}\right), x_{12}\left(t_{0}\right), x_{21}\left(t_{0}\right)\right) \mid x_{21}\left(t_{0}\right)=\mathcal{L}\left(x_{11}\left(t_{0}\right), x_{12}\left(t_{0}\right), t_{0}\right)\right. \\
& \left.=\frac{x_{11}\left(t_{0}\right)-x_{12}\left(t_{0}\right)+\sin t_{0}-e^{-t_{0}}+\cos t_{0}}{1+2 t_{0}} \forall x_{11}\left(t_{0}\right), x_{12}\left(t_{0}\right)\right\}
\end{aligned}
$$

Then, the index-2 semi explicit system will be as

$$
\begin{aligned}
\left(\begin{array}{l}
\dot{x}_{11} \\
\dot{x}_{12}
\end{array}\right) & =\left(\begin{array}{cc}
-1 & 1 \\
0 & 0
\end{array}\right)\left(\begin{array}{l}
x_{11} \\
x_{12}
\end{array}\right)+\left(\begin{array}{c}
0 \\
1+2 t
\end{array}\right) \mathcal{L}\left(x_{11}, x_{12}, t\right)+\left(\begin{array}{l}
1 \\
0
\end{array}\right) u(t) \\
0 & =\left(\begin{array}{ll}
1 & 1
\end{array}\right)\left(\begin{array}{l}
x_{11} \\
x_{12}
\end{array}\right)-\left(e^{-t}\right)+u(t) .
\end{aligned}
$$

And, as we mentioned in algorithm 5 for finding variational formulation,

$$
\begin{gathered}
F\left[x_{1}\right]=\frac{1}{2}\left\langle L_{1} x_{1}(t), L_{1} x_{1}(t)\right\rangle+\frac{1}{2}\left\langle L_{2} x_{1}(t), L_{2} x_{1}(t)\right\rangle+\frac{1}{2}\left\langle L_{3} x_{1}\left(t_{0}\right), L_{3} x_{1}\left(t_{0}\right)\right\rangle \\
-\left\langle\tilde{B}(t), L_{1} x_{1}(t)\right\rangle-\left\langle f(t), L_{2} x_{1}(t)\right\rangle-\left\langle f\left(t_{0}\right), L_{3} x_{1}\left(t_{0}\right)\right\rangle .
\end{gathered}
$$

where $L_{1} x_{1}(t)=\dot{x}_{1}+\tilde{A} x_{1}, \quad L_{2} x_{1}(t)=-A_{21} x_{1}, \quad L_{3} x_{1}\left(t_{0}\right)=-A_{21} x_{1}\left(t_{0}\right)$

$$
x_{1}=\left(x_{11}, x_{12}\right)^{T}, A_{11}=\left(\begin{array}{cc}
-1 & 1 \\
0 & 0
\end{array}\right), A_{12}=\left(\begin{array}{c}
0 \\
1+2 t
\end{array}\right), A_{21}=\left(\begin{array}{ll}
1 & 1
\end{array}\right) \text {, }
$$

$\tilde{A}=\left(A_{12}\left(A_{21} A_{12}\right)^{-1} A_{21} A_{11}-A_{11}\right), f(t)=-e^{t}+u(t)$,

$\tilde{B}=\left(1-A_{12}\left(A_{21} A_{12}\right)^{-1} A_{21}\right) B_{1} u-A_{12}\left(A_{21} A_{12}\right)^{-1}\left(e^{-t}-\right.$ cost $)$

$\mathcal{L}\left(x_{1}\right)=\frac{x_{11}-x_{12}+\sin t-e^{-t}+\cos t}{1+2 t}$ are defined with the class $\omega^{0}$.

The variational formulation with the class of consistent initial condition is defined as:

Now, we set

$$
\begin{aligned}
F\left[x_{1}\right]=\frac{1}{2} \int_{0}^{1}\left[\left[\dot{x}_{1}+\tilde{A} x_{1}\right]^{T}\left[\dot{x}_{1}+\tilde{A} x_{1}\right]-2 \tilde{B}^{T}\left[\dot{x}_{1}+\tilde{A} x_{1}\right]+\left[-A_{21} x_{1}\right]^{T}\left[-A_{21} x_{1}\right]\right. \\
-2[f(t)]^{T}\left[-A_{21} x_{1}\right]+\left[-A_{21} x_{1}\left(t_{0}\right)\right]^{T}\left[-A_{21} x_{1}\left(t_{0}\right)\right] \\
\left.-2\left[-\left(e^{-t_{0}}\right)+u\left(t_{0}\right)\right]^{T}\left[-A_{21} x_{1}\left(t_{0}\right)\right]\right] d t
\end{aligned}
$$

$$
\begin{aligned}
& x_{11}(t)=\sum_{i=0}^{m_{1}} a_{i}^{1} H_{i}^{1}(t), H_{i}^{1}(t)=t^{i}, i=0, \ldots, m_{1} ; m_{1}=5, \\
& x_{12}(t)=\sum_{i=0}^{m_{2}} a_{i}^{2} H_{i}^{2}(t), H_{i}^{2}(t)=t^{i}, i=0, \ldots, m_{2} ; m_{2}=5
\end{aligned}
$$

and $H_{i}^{1}, H_{i}^{2}$ are linearly independent-bases functions that are vanished on $\omega^{0}$. 
We estimate $F\left(\overrightarrow{a^{1}}, \overrightarrow{a^{2}}\right)=F\left(a_{0}^{1}, a_{1}^{1}, a_{2}^{1}, \ldots, a_{5}^{1}, a_{0}^{2}, a_{1}^{2}, a_{2}^{2}, \ldots, a_{5}^{2}\right)$, and by taking $\frac{\partial F}{\partial \overrightarrow{a^{1}}}=0$, and $\frac{\partial F}{\partial \overrightarrow{a^{2}}}=0$, this gives $A\left(\frac{\overrightarrow{a^{1}}}{\overrightarrow{a^{2}}}\right)=B$, hence the approximate solution $\left(x_{11}(t), x_{12}(t), x_{21}(t)\right)$ is obtained.

The numerical results of the unknown coefficients of linear algebraic system were found to be:

$a_{0}^{1}=1, a_{1}^{1}=-0.99997, a_{2}^{1}=0.49967, a_{3}^{1}=-0.16510, a_{4}^{1}=0.03840, a_{5}^{1}=-0.00511$ $, a_{0}^{2}=0, a_{1}^{2}=0.99998, a_{2}^{2}=0.00020, a_{3}^{3}=-0.16757, a_{4}^{2}=0.00162, a_{5}^{2}=0.00723$.

And The exact solution that taken from [1] is

$x_{11}=e^{-t}, \quad x_{12}=\sin t, \quad x_{21}(t)=\frac{\text { cost }}{1+2 t}$ for a given $u(t)=-$ sint .

Then one can shows the comparison between the proposed solution and the exact solution in tables 5.1 and 5.2.

Table 5.1-Comparison between differential states in the proposed method and exact solution

\begin{tabular}{c|cccccc}
\hline & $\begin{array}{c}\boldsymbol{x}_{\mathbf{1 1}} \\
\text { Propose } \\
\text { method }\end{array}$ & $\begin{array}{c}\boldsymbol{x}_{\mathbf{1 1}} \\
\text { Exact } \\
\text { Sol. }\end{array}$ & Abs. error & $\begin{array}{c}\boldsymbol{x}_{\mathbf{1 2}} \\
\text { Propose } \\
\text { method }\end{array}$ & $\begin{array}{c}\boldsymbol{x}_{\mathbf{1 2}} \\
\text { Exact } \\
\text { Sol. }\end{array}$ & Abs. error \\
\hline $\mathbf{0}$ & 1 & 1 & 0 & 0 & 0 & 0 \\
$\mathbf{0 . 1}$ & 0.9048 & 0.9048 & $1496 \times 10^{-7}$ & 0.0998 & 0.0998 & $1299 \times 10^{-7}$ \\
$\mathbf{0 . 2}$ & 0.8187 & 0.8187 & $5443 \times 10^{-7}$ & 0.1986 & 0.1987 & $3842 \times 10^{-7}$ \\
$\mathbf{0 . 3}$ & 0.7408 & 0.7408 & $3639 \times 10^{-7}$ & 0.2955 & 0.2955 & $3052 \times 10^{-7}$ \\
$\mathbf{0 . 4}$ & 0.6703 & 0.6703 & $3007 \times 10^{-7}$ & 0.3894 & 0.3894 & $1859 \times 10^{-7}$ \\
$\mathbf{0 . 5}$ & 0.6065 & 0.6065 & $6213 \times 10^{-7}$ & 0.4794 & 0.4794 & $4818 \times 10^{-7}$ \\
$\mathbf{0 . 6}$ & 0.5488 & 0.5488 & $2583 \times 10^{-7}$ & 0.5646 & 0.5646 & $2461 \times 10^{-7}$ \\
$\mathbf{0 . 7}$ & 0.4965 & 0.4966 & $3840 \times 10^{-7}$ & 0.6442 & 0.6442 & $2770 \times 10^{-7}$ \\
$\mathbf{0 . 8}$ & 0.4493 & 0.4493 & $5108 \times 10^{-7}$ & 0.7173 & 0.7174 & $4324 \times 10^{-7}$ \\
$\mathbf{0 . 9}$ & 0.4065 & 0.4066 & $1605 \times 10^{-7}$ & 0.7833 & 0.7833 & $1139 \times 10^{-7}$ \\
$\mathbf{1}$ & 0.3678 & 0.3679 & $3043 \times 10^{-10}$ & 0.8414 & 0.8415 & $2775 \times 10^{-10}$
\end{tabular}

Table 5.2-Comparison between equality constraint states in the proposed method and exact solution

\begin{tabular}{c|ccc}
\hline $\mathbf{t}$ & $\begin{array}{c}\boldsymbol{x}_{\mathbf{2 1}} \\
\text { Prposed } \\
\text { method }\end{array}$ & $\begin{array}{c}\boldsymbol{x}_{\mathbf{2 1}} \\
\text { Exact } \\
\text { Sol. }\end{array}$ & Abs. error \\
\hline $\mathbf{0}$ & 1 & 1 & 0 \\
$\mathbf{0 . 1}$ & 0.8291 & 0.8292 & $2329 \times 10^{-7}$ \\
$\mathbf{0 . 2}$ & 0.7000 & 0.7000 & $6633 \times 10^{-7}$ \\
$\mathbf{0 . 3}$ & 0.5970 & 0.5971 & $4182 \times 10^{-7}$ \\
$\mathbf{0 . 4}$ & 0.5117 & 0.5117 & $2703 \times 10^{-7}$ \\
$\mathbf{0 . 5}$ & 0.4387 & 0.4388 & $5516 \times 10^{-7}$ \\
$\mathbf{0 . 6}$ & 0.3751 & 0.3752 & $2293 \times 10^{-7}$ \\
$\mathbf{0 . 7}$ & 0.3186 & 0.3187 & $2754 \times 10^{-7}$ \\
$\mathbf{0 . 8}$ & 0.2679 & 0.2680 & $3628 \times 10^{-7}$ \\
$\mathbf{0 . 9}$ & 0.2220 & 0.2220 & $9804 \times 10^{-7}$ \\
$\mathbf{1}$ & 0.1801 & 0.1801 & $1939 \times 10^{-10}$ \\
\hline
\end{tabular}

There is another way to test the accuracy of the solution, without knowing the exact solution, by using $L_{2}$-norm and substituting the $a_{i}^{j}$ values in 


$$
\begin{aligned}
& x_{11}(t)=\sum_{i=0}^{m_{1}} a_{i}^{1} H_{i}^{1}(t), H_{i}^{1}(t)=t^{i}, i=0, \ldots, m_{1} ; m_{1}=5, \\
& x_{12}(t)=\sum_{i=0}^{m_{2}} a_{i}^{2} H_{i}^{2}(t), H_{i}^{2}(t)=t^{i}, i=0, \ldots, m_{2} ; m_{2}=5
\end{aligned}
$$

So for differential equation, one can check the accuracy as follows

$$
\begin{aligned}
\left\|\dot{x}_{1}-A_{11} x_{1}-A_{12} x_{2}-B_{1} u\right\|_{2}= & {\left[\int _ { 0 } ^ { 1 } \left[\left|\dot{x}_{11}+x_{11}-x_{12}+\sin t\right|^{2}+\mid \dot{x}_{12}-x_{11}+x_{12}-\right.\right.} \\
& \text { sint } \left.\left.+e^{-t}-\left.\cos t\right|^{2}\right] d t\right]^{1 / 2} \\
= & 3 \times 10^{-5}
\end{aligned}
$$

where $x_{1}=\left(x_{11}, x_{12}\right)^{T}$, and for equality algebraic constraint

$$
\left\|A_{21} x_{1}-e^{-t}+u\right\|_{2}=\left[\int_{0}^{1}\left|x_{11}+x_{12}-e^{-t}-\sin t\right|^{2} d t\right]^{1 / 2}=2 \times 10^{-7}
$$

And for consistency condition

$$
\left\|A_{21} x_{1}\left(t_{0}\right)-e^{-t_{0}}+u\left(t_{0}\right)\right\|_{2}=\left[\int_{0}^{1}\left|x_{11}\left(t_{0}\right)+x_{12}\left(t_{0}\right)-e^{-t_{0}}-\sin t_{0}\right|^{2} d t\right]^{1 / 2}=0
$$

The $L_{2}$ norm errors (23)-(25) explain the overall error of satisfying the equations (10)-(12), $\forall t \in\left[t_{0}, t_{f}\right]=[0,1]$, for each equation, where the parameterizations (21),(22) are suggested. The approximate and exact solution to the differential-equality states are showed in Figure 5.1 .

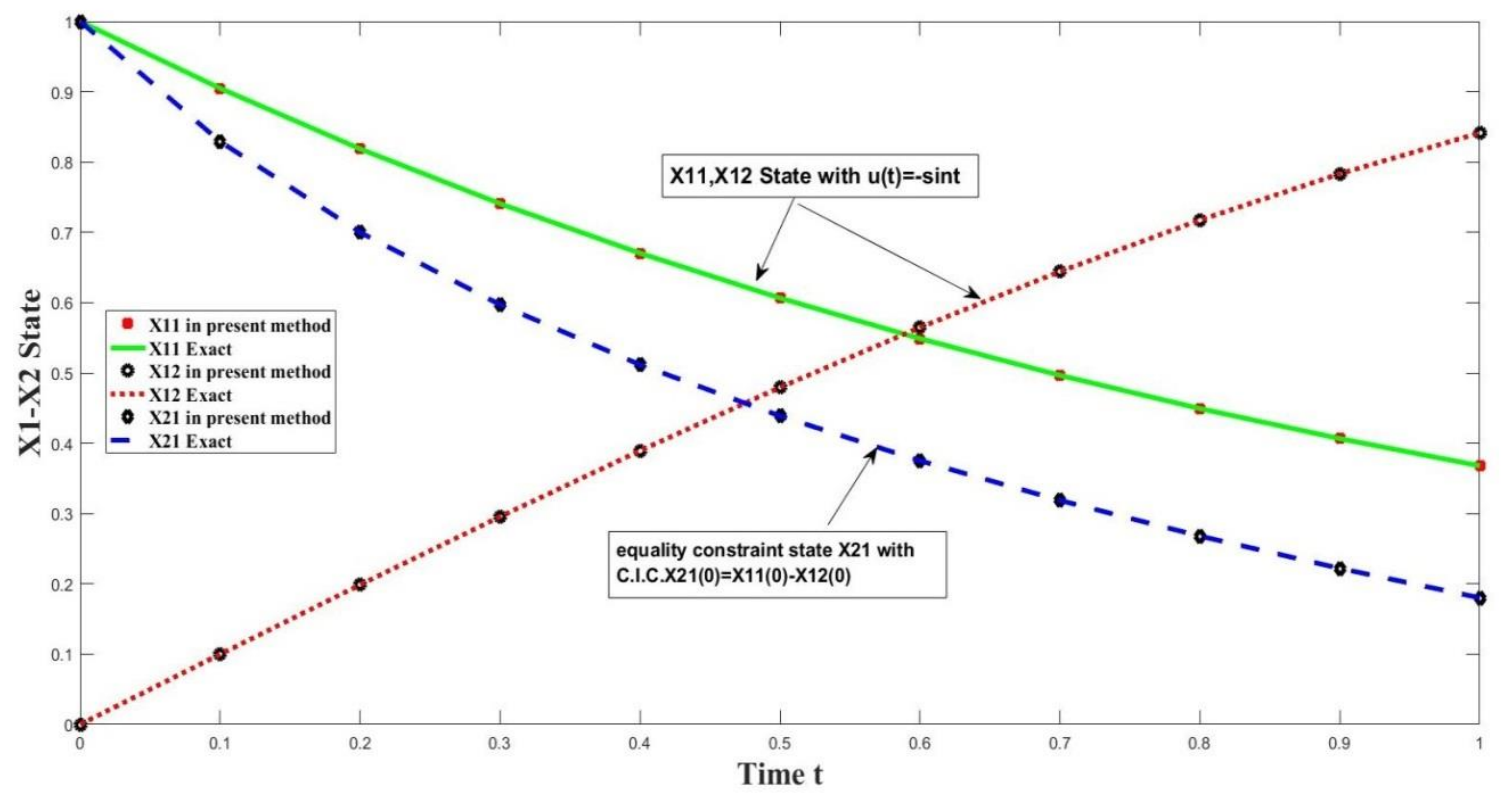

Figure 5.1- Differential-equality states and exact solution of index 2 linear time-varying DAEs with given $u(t)=-$ sint.

Example 5.2: (The algebraic equation appears as a system of equations) Consider the linear time invariant descriptor system

$$
\begin{gathered}
\dot{x}_{11}=x_{11}+2 x_{21}(t)+x_{22}(t)+u(t)+f_{11}(t) \\
\dot{x}_{12}=2 x_{11}(t)+x_{12}(t)+2 x_{22}(t)+f_{12}(t) \\
0=x_{11}(t)-u(t)+f_{21}(t) \\
0=x_{12}(t)+f_{22}(t)
\end{gathered}
$$

with

$$
\begin{aligned}
& f_{11}(t)=-t^{5}+3 t^{4}-t^{3}-2 t^{2}-1-u(t), \\
& f_{12}(t)=2 t^{5}-t^{4}+t^{3}-t^{2}-4, \\
& f_{21}(t)=-1-t^{2}-t^{5}+u(t), \\
& f_{22}(t)=-2-t^{3}-t^{4}, \text { where } \in[0,1] .
\end{aligned}
$$

This system is equivalent to the following differential-algebraic system

$$
\dot{x}_{1}=A_{11} x_{1}+A_{12} x_{2}+B_{1} u(t)+f_{1}
$$




$$
0=A_{21} x_{1}+B_{2} u(t)+f_{2} .
$$

where $x_{1}=\left(x_{11}, x_{12}\right)^{T}, x_{2}=\left(x_{21}, x_{22}\right)^{T}, A_{11}=\left(\begin{array}{ll}1 & 0 \\ 2 & 1\end{array}\right), A_{12}=\left(\begin{array}{ll}2 & 1 \\ 0 & 2\end{array}\right), A_{21}=\left(\begin{array}{ll}1 & 0 \\ 0 & 1\end{array}\right)$, $f_{1}=\left(f_{11}, f_{12}\right)^{T}, f_{2}=\left(f_{21}, f_{22}\right)^{T}, B_{1}=\left(\begin{array}{ll}1 & 0\end{array}\right)^{T}, B_{1}=\left(\begin{array}{ll}-1 & 0\end{array}\right)^{T}, u(t) \in \Delta_{u}$, where $\Delta_{u}$ is the class of admissible control.

If we differentiate the equality constraint with respect to $t$, one can get

$$
\begin{gathered}
0=A_{21} \dot{x}_{1}+B_{2} \dot{u}(t)+\dot{f}_{2} \\
0=A_{21}\left(A_{11} x_{1}+A_{12} x_{2}+B_{1} u(t)+f_{1}\right)+B_{2} \dot{u}(t)+\dot{f}_{2}
\end{gathered}
$$

Since $\left(A_{21} A_{12}\right)$ is nonsingular, then it is possible to rewrite our system as

$$
\begin{aligned}
\dot{x}_{1}= & \left(A_{11}-A_{12}\left(\left(A_{21} A_{12}\right)^{-1}\left(A_{21} A_{11}\right)\right)\right) x_{1}+\left(B_{1}-A_{12}\left(A_{21} A_{12}\right)^{-1} A_{21} B_{1}\right) u(t) \\
& \quad+\left(1-A_{12}\left(A_{21} A_{12}\right)^{-1} A_{21}\right) f_{1}-A_{12}\left(A_{21} A_{12}\right)^{-1} B_{2} \dot{u}(t)-A_{12}\left(A_{21} A_{12}\right)^{-1} \dot{f}_{2} \\
x_{2}= & \mathcal{L}\left(x_{1}, u, \dot{u}, f_{1}, \dot{f}_{2}, t\right) \\
= & -\left(A_{21} A_{12}\right)^{-1}\left(\left(A_{21} A_{11}\right) x_{1}+A_{21} B_{1} u(t)+A_{21} f_{1}(t)+B_{2} \dot{u}(t)+\dot{f}_{2}(t)\right) \\
= & \left(\begin{array}{c}
\frac{3 t^{4}}{4}-\frac{t^{3}}{4}+t-\frac{x_{12}}{4}-\frac{1}{2} \\
t^{5}+\frac{t^{4}}{2}+\frac{3 t^{3}}{2}+2 t^{2}-x_{11}-\frac{x_{12}}{2}+2
\end{array}\right) \text { defined with the class } \omega^{0} .
\end{aligned}
$$

The class of consistency initial condition is

$$
\begin{aligned}
\omega^{0}= & \left\{\left(x_{11}\left(t_{0}\right), x_{12}\left(t_{0}\right), x_{21}\left(t_{0}\right), x_{22}\left(t_{0}\right)\right) \mid x_{21}\left(t_{0}\right)=\frac{x_{12}\left(t_{0}\right)}{4}-\frac{1}{2}\right. \\
& \left., x_{22}\left(t_{0}\right)=2-x_{11}(0)-\frac{x_{12}(0)}{2} \forall x_{11}(0), x_{12}(0)\right\} .
\end{aligned}
$$

The variational formulation with the class of consistent initial condition is defined as

where

$$
\begin{aligned}
F\left[x_{1}\right]=\frac{1}{2} \int_{0}^{1}[ & {\left[\dot{x}_{1}+\tilde{A} x_{1}\right]^{T}\left[\dot{x}_{1}+\tilde{A} x_{1}\right]-2 \tilde{B}^{T}\left[\dot{x}_{1}+\tilde{A} x_{1}\right]+\left[-A_{21} x_{1}\right]^{T}\left[-A_{21} x_{1}\right] } \\
& -2\left[B_{2} u+f_{2}(t)\right]^{T}\left[-A_{21} x_{1}\right]+\left[-A_{12} x_{1}^{0}\right]^{T}\left[A_{12} x_{1}^{0}\right] \\
& \left.-2\left[B_{2} u\left(t_{0}\right)+f_{2}\left(t_{0}\right)\right]^{T}\left[A_{12} x_{1}^{0}\right]\right] d t
\end{aligned}
$$

$$
\begin{gathered}
\tilde{A}=\left(A_{12}\left(A_{21} A_{12}\right)^{-1} A_{21} A_{11}-A_{11}\right) \\
\tilde{B}=\left(1-A_{12}\left(A_{21} A_{12}\right)^{-1} A_{21}\right) B_{1} u-\left(1-A_{12}\left(A_{21} A_{12}\right)^{-1} A_{21}\right) f_{1}-A_{12}\left(A_{21} A_{12}\right)^{-1} B_{2} \dot{u}(t) \\
\quad-A_{12}\left(A_{21} A_{12}\right)^{-1} \dot{f}_{2} .
\end{gathered}
$$

Now, we set

$$
\begin{aligned}
x_{11}(t) & =\sum_{i=0}^{m_{1}} a_{i}^{1} H_{i}^{1}(t), H_{i}^{1}(t)=t^{i}, i=0, \ldots, m_{1} ; m_{1}=5, \\
x_{12}(t) & =\sum_{i=0}^{m_{2}} a_{i}^{2} H_{i}^{2}(t), H_{i}^{2}(t)=t^{i}, i=0, \ldots, m_{2} ; m_{2}=5
\end{aligned}
$$

and $H_{i}^{1}, H_{i}^{2}$ are linearly independent-base functions that are vanished on $\omega^{0}$, which leads to

$$
x_{2}(t)=\mathcal{L}\left(x_{11}, x_{12}, t\right)=\mathcal{L}\left(\sum_{i=0}^{m_{1}} a_{i}^{1} H_{i}^{1}(t), \sum_{i=0}^{m_{2}} a_{i}^{2} H_{i}^{2}(t), t\right)
$$

then $F\left(\overrightarrow{a^{1}}, \overrightarrow{a^{2}}\right)=F\left(a_{0}^{1}, a_{1}^{1}, a_{2}^{1}, \ldots, a_{5}^{1}, a_{0}^{2}, a_{1}^{2}, a_{2}^{2}, \ldots, a_{5}^{2}\right)$, and by taking $\frac{\partial F}{\partial \overrightarrow{a^{1}}}=0$, and $\frac{\partial F}{\partial \overrightarrow{a^{2}}}=0$, this gives $A\left(\frac{\overrightarrow{a^{1}}}{a^{2}}\right)=B$, hence the numerical solution for $a^{1}, a^{2}$ using the proposed technique is found as: 
where the exact answer is obtained as:

$$
\begin{aligned}
& a_{0}^{1}=1, a_{1}^{1}=0, a_{2}^{1}=1, a_{3}^{1}=0, a_{4}^{1}=0, a_{5}^{1}=1, \\
& a_{0}^{2}=2, a_{1}^{2}=0, a_{2}^{2}=0, a_{3}^{3}=1, a_{4}^{2}=1, a_{5}^{2}=0,
\end{aligned}
$$

$$
x_{11}=1+t^{2}+t^{5}, \quad x_{12}=2+t^{3}+t^{4}, \quad x_{21}=t+t^{4}, \quad x_{22}=t^{2}+t^{3} .
$$

The numerical results used in the proposed technique and the comparisons with given exact solutions are shown in following tables.

Table 5.3-Comparisons among differential states in the proposed method and exact solutions

\begin{tabular}{c|cccccc}
\hline $\mathbf{t}$ & $\begin{array}{c}\boldsymbol{x}_{\mathbf{1 1}} \\
\text { Proposed } \\
\text { method }\end{array}$ & $\begin{array}{c}\boldsymbol{x}_{\mathbf{1 1}} \\
\text { exact }\end{array}$ & $\begin{array}{c}\text { Abs. } \\
\text { error }\end{array}$ & $\begin{array}{c}\boldsymbol{x}_{\mathbf{1 2}} \\
\text { Proposed } \\
\text { method }\end{array}$ & $\begin{array}{c}\boldsymbol{x}_{\mathbf{1 2}} \\
\text { exact }\end{array}$ & $\begin{array}{c}\text { Abs. } \\
\text { error }\end{array}$ \\
\hline $\mathbf{0}$ & 1 & 1 & 0 & 2 & 2 & 0 \\
$\mathbf{0 . 1}$ & 1.0100 & 1.0100 & $7 \times 10^{-13}$ & 2.0011 & 2.0011 & 0 \\
$\mathbf{0 . 2}$ & 1.0403 & 1.0403 & 0 & 2.0096 & 2.0096 & 0 \\
$\mathbf{0 . 3}$ & 1.0924 & 1.0924 & $1 \times 10^{-13}$ & 2.0351 & 2.0351 & 0 \\
$\mathbf{0 . 4}$ & 1.1702 & 1.1702 & 0 & 2.0896 & 2.0896 & 0 \\
$\mathbf{0 . 5}$ & 1.2812 & 1.2813 & 0 & 2.1875 & 2.1875 & 0 \\
$\mathbf{0 . 6}$ & 1.4377 & 1.4378 & 0 & 2.3456 & 2.3456 & 0 \\
$\mathbf{0 . 7}$ & 1.6580 & 1.6581 & $6 \times 10^{-14}$ & 2.5831 & 2.5831 & 0 \\
$\mathbf{0 . 8}$ & 1.9676 & 1.9677 & 0 & 2.9216 & 2.9216 & 0 \\
$\mathbf{0 . 9}$ & 2.4004 & 2.4005 & $1 \times 10^{-14}$ & 3.3851 & 3.3851 & 0 \\
$\mathbf{1}$ & 3 & 3 & 0 & 4 & 4 & 0 \\
\hline
\end{tabular}

Table 5.4-Comparison among equality state in proposed method and exact solutions

\begin{tabular}{c|cccccc}
\hline $\mathbf{t}$ & $\begin{array}{c}\boldsymbol{x}_{\mathbf{2 1}} \\
\text { Proposed } \\
\text { method }\end{array}$ & $\begin{array}{c}\boldsymbol{x}_{\mathbf{2}} \\
\text { exact }\end{array}$ & Abs. error & $\begin{array}{c}\boldsymbol{x}_{22} \\
\text { Proposed } \\
\text { method }\end{array}$ & $\begin{array}{c}\boldsymbol{x}_{22} \\
\text { exact }\end{array}$ & $\begin{array}{c}\text { Abs. } \\
\text { error }\end{array}$ \\
\hline $\mathbf{0}$ & 0 & 0 & 0 & 0 & 0 & 0 \\
$\mathbf{0 . 1}$ & 0.1001 & 0.1001 & 0 & 0.011 & 0.011 & 0 \\
$\mathbf{0 . 2}$ & 0.2016 & 0.2016 & 0 & 0.048 & 0.048 & 0 \\
$\mathbf{0 . 3}$ & 0.3081 & 0.3081 & 0 & 0.117 & 0.117 & 0 \\
$\mathbf{0 . 4}$ & 0.4256 & 0.4256 & 0 & 0.224 & 0.224 & 0 \\
$\mathbf{0 . 5}$ & 0.5625 & 0.5625 & 0 & 0.375 & 0.375 & 0 \\
$\mathbf{0 . 6}$ & 0.7296 & 0.7296 & 0 & 0.576 & 0.576 & 0 \\
$\mathbf{0 . 7}$ & 0.9401 & 0.9401 & 0 & 0.833 & 0.833 & 0 \\
$\mathbf{0 . 8}$ & 1.2096 & 1.2096 & 0 & 1.152 & 1.152 & 0 \\
$\mathbf{0 . 9}$ & 1.5561 & 1.5561 & 0 & 1.539 & 1.539 & 0 \\
\hline
\end{tabular}




\begin{tabular}{l|llllll}
\hline 1 & 2 & 2 & 0 & 2 & 0 \\
\hline
\end{tabular}

To test the accuracy of the solution presented by the present method, the $L_{2}$-norm is examined.

For equality constraint, we have

$$
\left\|A_{21} x_{1}-e^{-t}+u\right\|_{2}=\left[\int_{0}^{1}\left|x_{11}+x_{12}-e^{-t}-\sin t\right|^{2} d t\right]^{1 / 2}=0
$$

This criterion represents a good test to show the extent to which the approximate solution matches the exact solution, and thus indicates the accuracy of the method used.

The differential-equality states are illustrated in Figure 5.2.

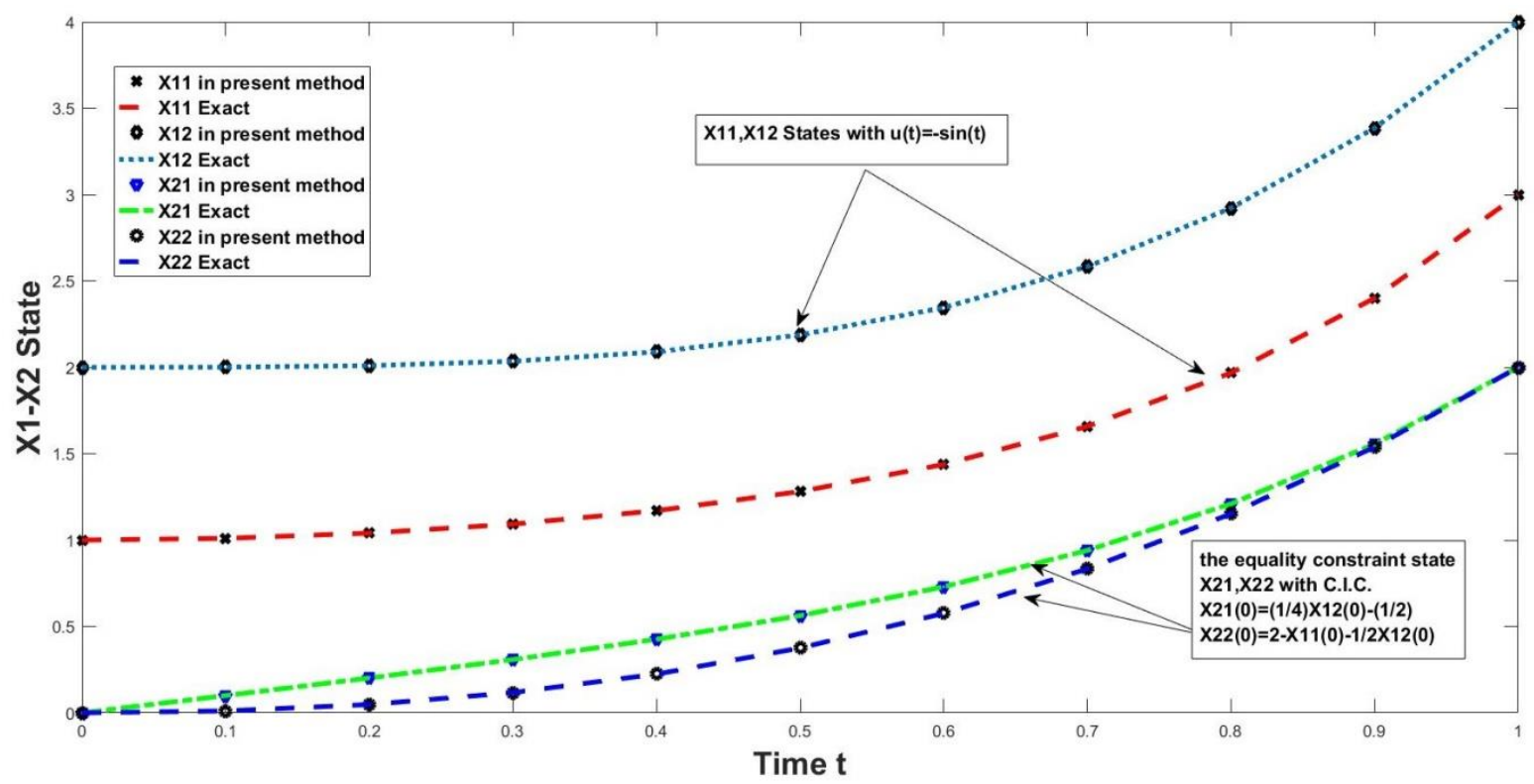

Figure 5.2- Differential-equality states and exact solution with $u(t)=-$ sint. C.I.C. is consistent initial condition.

Example 5.3 (Descriptor index of two control model)

Consider the following linear mechanical system

with $E=\left[\begin{array}{ccc}I & 0 & 0 \\ 0 & \mathcal{M} & 0 \\ 0 & 0 & 0\end{array}\right], A=\left[\begin{array}{ccc}0 & I & I \\ -\mathcal{K} & -\mathcal{D} & \mathcal{J} \\ \mathcal{H} & G & 0\end{array}\right], B=\left[\begin{array}{l}0 \\ \mathcal{L} \\ 0\end{array}\right], x=\left[\begin{array}{c}Z \\ \dot{Z} \\ \mu\end{array}\right]$

where the matrices in the table below represent the respective models.

\begin{tabular}{cc}
\hline Matrix & Represent in mechanical model \\
\hline $\boldsymbol{Z} \in \boldsymbol{R}^{\boldsymbol{n}}$ & the displacement vector \\
$\boldsymbol{\mu} \in \boldsymbol{R}^{\boldsymbol{q}}$ & the vector of lagrangian multiplier \\
$\boldsymbol{u}$ & the known input force \\
$\mathcal{M}$ & the inertial matrix \\
$\mathcal{D}$ & the damping matrix \\
$\mathcal{K}$ & the stiffness matrix \\
$\mathcal{L}$ & matrix of force distribution \\
$G, \mathcal{H}$ & the coefficient matrices \\
\hline
\end{tabular}

All these matrices are known with appropriate dimensions. For more detail about this mechanical model, see [14].

Now, based on these matrices the semi explicit descriptor system can be rewritten as 
$\left[\begin{array}{lllll}1 & 0 & 0 & 0 & 0 \\ 0 & 1 & 0 & 0 & 0 \\ 0 & 0 & 1 & 0 & 0 \\ 0 & 0 & 0 & 1 & 0 \\ 0 & 0 & 0 & 0 & 0\end{array}\right] x=\left[\begin{array}{ccccc}0 & 0 & 1 & 0 & 1 \\ 0 & 0 & 0 & 1 & 1 \\ -2 & 0 & -1 & -1 & 1 \\ 0 & -1 & -1 & -1 & 1 \\ 1 & 1 & 0 & 0 & 0\end{array}\right] x+\left[\begin{array}{c}0 \\ 0 \\ -1 \\ 1 \\ 0\end{array}\right] u(t)+\left[\begin{array}{c}1+t \\ t \\ 0 \\ 0 \\ t^{2}+t\end{array}\right]$

This system needs to be transform firstly into DAEs, as mentioned previously in section 3 , with $\operatorname{rank}(E)=4<n$. Then, a singular value decomposition is used to calculate $U$ as an orthonormal eigenvectors matrix of $E E^{T}$ and $V$ as an orthonormal eigenvectors matrix of $E^{T} E$.

Set $\mathbb{P}=V=\left[\begin{array}{lllll}0 & 0 & 0 & 1 & 0 \\ 1 & 0 & 0 & 0 & 0 \\ 0 & 1 & 0 & 0 & 0 \\ 0 & 0 & 1 & 0 & 0 \\ 0 & 0 & 0 & 0 & 1\end{array}\right], \quad \mathbb{Q}=U^{-1}=\left[\begin{array}{lllll}0 & 1 & 0 & 0 & 0 \\ 0 & 0 & 1 & 0 & 0 \\ 0 & 0 & 0 & 1 & 0 \\ 1 & 0 & 0 & 0 & 0 \\ 0 & 0 & 0 & 0 & 1\end{array}\right]$

Now $\mathbb{Q} E \mathbb{P}=\left[\begin{array}{lllll}1 & 0 & 0 & 0 & 0 \\ 0 & 1 & 0 & 0 & 0 \\ 0 & 0 & 1 & 0 & 0 \\ 0 & 0 & 0 & 1 & 0 \\ 0 & 0 & 0 & 0 & 0\end{array}\right] \Rightarrow \Sigma=\mathrm{I}_{4 \times 4}=\Sigma^{-1}, \quad \mathbb{P}^{-1} x=\left[\begin{array}{l}x_{11} \\ x_{12} \\ x_{13} \\ x_{14} \\ x_{21}\end{array}\right]$

$$
\mathbb{Q} A \mathbb{P}=\left[\begin{array}{ccccc}
0 & 0 & 1 & 0 & 1 \\
0 & -1 & -1 & -2 & 1 \\
-1 & -1 & -1 & 0 & 1 \\
0 & 1 & 0 & 0 & 1 \\
1 & 0 & 0 & 1 & 0
\end{array}\right], \quad \mathbb{Q} B=\left[\begin{array}{c}
0 \\
-1 \\
1 \\
0 \\
0
\end{array}\right], \quad \mathbb{Q} f=\left[\begin{array}{c}
t \\
0 \\
0 \\
t+1 \\
t^{2}+t
\end{array}\right]
$$

Under the transformation $(\mathbb{Q}, \mathbb{P})$, the mechanical system will be differential algebraic control system as:

$$
\begin{gathered}
\dot{x}_{1}=A_{11} x_{1}+A_{12} x_{2}+B_{1} u+f_{1} \\
0=A_{21} x_{1}+f_{2}
\end{gathered}
$$

with $A_{11}=\left[\begin{array}{crrr}0 & 0 & 1 & 0 \\ 0 & -1 & -1 & -2 \\ -1 & -1 & -1 & 0 \\ 0 & 1 & 0 & 0\end{array}\right], A_{12}=\left[\begin{array}{l}1 \\ 1 \\ 1 \\ 1\end{array}\right], B_{1}=\left[\begin{array}{c}0 \\ -1 \\ 1 \\ 0\end{array}\right], A_{21}=\left[\begin{array}{llll}1 & 0 & 0 & 1\end{array}\right]$,

$$
f_{1}=\left[\begin{array}{c}
t \\
0 \\
0 \\
1+t
\end{array}\right], f_{2}=t^{2}+t, x_{1}=\left[\begin{array}{c}
x_{11} \\
x_{12} \\
x_{13} \\
x_{14}
\end{array}\right], x_{2}=x_{21}
$$

which is an index-2 system. By differentiating the equality constraint with respect to $t$ to estimate $x_{2}$, and since $\left(A_{21} A_{12}\right)$ invertible matrix, then

$$
\begin{aligned}
& x_{2}=-\left(A_{21} A_{12}\right)^{-1}\left[A_{21} A_{11} x_{1}+A_{21} B_{1} u+A_{21} f_{1}+B_{2} \dot{u}+\dot{f}_{2}\right] \\
& x_{2}=-2 t-\frac{x_{12}+x_{13}+1}{2} \text { is defined with the class } \omega^{0} .
\end{aligned}
$$

And the class of consistency initial condition is

$$
\begin{aligned}
\omega^{0}=\left\{\left(x_{11}\left(t_{0}\right)\right.\right. & \left., x_{12}\left(t_{0}\right), x_{13}\left(t_{0}\right), x_{14}\left(t_{0}\right), x_{21}\left(t_{0}\right)\right) \mid x_{21}\left(t_{0}\right) \\
& \left.=\mathcal{L}\left(x_{11}\left(t_{0}\right), x_{12}\left(t_{0}\right), x_{13}\left(t_{0}\right), x_{14}\left(t_{0}\right), t_{0}\right) \forall x_{12}\left(t_{0}\right), x_{13}\left(t_{0}\right)\right\} .
\end{aligned}
$$

The variational function with the class of consistent initial condition is defined as

where

$$
\begin{aligned}
F\left[x_{1}\right]=\frac{1}{2} \int_{0}^{1}\left[\left[\dot{x}_{1}+\tilde{A} x_{1}\right]^{T}\left[\dot{x}_{1}+\tilde{A} x_{1}\right]-2 \tilde{B}^{T}\left[\dot{x}_{1}+\tilde{A} x_{1}\right]+\left[-A_{21} x_{1}\right]^{T}\left[-A_{21} x_{1}\right]\right. \\
-2\left[B_{2} u+f_{2}(t)\right]^{T}\left[-A_{21} x_{1}\right]+\left[-A_{21} x_{1}\left(t_{0}\right)\right]^{T}\left[-A_{21} x_{1}\left(t_{0}\right)\right] \\
\left.-2\left[f_{2}\left(t_{0}\right)\right]^{T}\left[-A_{21} x_{1}\left(t_{0}\right)\right]\right] d t
\end{aligned}
$$

$\tilde{A}=\left(A_{12}\left(A_{21} A_{12}\right)^{-1} A_{21} A_{11}-A_{11}\right)$, 


$$
\begin{gathered}
\tilde{B}=\left(1-A_{12}\left(A_{21} A_{12}\right)^{-1} A_{21}\right) B_{1} u-\left(1-A_{12}\left(A_{21} A_{12}\right)^{-1} A_{21}\right) f_{1}-A_{12}\left(A_{21} A_{12}\right)^{-1} B_{2} \dot{u}(t) \\
-A_{12}\left(A_{21} A_{12}\right)^{-1} \dot{f}_{2}
\end{gathered}
$$

Now, we set

$$
\begin{aligned}
x_{11}(t) & =\sum_{i=0}^{m_{1}} a_{i}^{1} H_{i}^{1}(t), H_{i}^{1}(t)=t^{i}, i=0, \ldots, m_{1} ; m_{1}=5, \\
x_{12}(t) & =\sum_{i=0}^{m_{2}} a_{i}^{2} H_{i}^{2}(t), H_{i}^{2}(t)=t^{i}, i=0, \ldots, m_{2} ; m_{2}=5 \\
x_{13}(t) & =\sum_{i=0}^{m_{3}} a_{i}^{3} H_{i}^{3}(t), H_{i}^{3}(t)=t^{i}, i=0, \ldots, m_{3} ; m_{3}=5 \\
x_{14}(t) & =\sum_{i=0}^{m_{4}} a_{i}^{4} H_{i}^{4}(t), H_{i}^{4}(t)=t^{i}, i=0, \ldots, m_{4} ; m_{4}=5
\end{aligned}
$$

and $H_{i}^{1}, H_{i}^{2}, H_{i}^{3}, H_{i}^{4}$ are linearly independent-base functions that are vanished on $\omega^{0}$, which leads to

$$
\begin{aligned}
x_{21}(t) & =\mathcal{L}\left(x_{11}, x_{12}, x_{13}, x_{14}, t\right) \\
& =\mathcal{L}\left(\sum_{i=0}^{m_{1}} a_{i}^{1} H_{i}^{1}(t), \sum_{i=0}^{m_{2}} a_{i}^{2} H_{i}^{2}(t), \sum_{i=0}^{m_{3}} a_{i}^{3} H_{i}^{3}(t), \sum_{i=0}^{m_{4}} a_{i}^{4} H_{i}^{4}(t), t\right)
\end{aligned}
$$

And $F\left(\overrightarrow{a^{1}}, \overrightarrow{a^{2}}, \overrightarrow{a^{3}}, \overrightarrow{a^{4}}\right), \overrightarrow{a^{1}}=\left(a_{0}^{1}, a_{1}^{1}, a_{2}^{1}, \ldots, a_{m_{1}}^{1}\right), \overrightarrow{a^{2}}=\left(a_{0}^{2}, a_{1}^{2}, a_{2}^{2}, \ldots, a_{m_{2}}^{2}\right)$

$$
\overrightarrow{a^{3}}=\left(a_{0}^{3}, a_{1}^{3}, a_{2}^{3}, \ldots, a_{m_{3}}^{3}\right), \overrightarrow{a^{4}}=\left(a_{0}^{4}, a_{1}^{4}, a_{2}^{4}, \ldots, a_{m_{4}}^{4}\right)
$$

Since it is a quadratic functional form, for finding the critical point ,this leads to the linear algebraic equation $A\left(\begin{array}{l}\overrightarrow{a^{1}} \\ \frac{a^{2}}{\overrightarrow{a^{3}}} \\ \frac{\overrightarrow{a^{4}}}{\vec{a}}\end{array}\right)=B$, which is solvable directly for $\left(\begin{array}{l}\frac{\overrightarrow{a^{1}}}{a^{2}} \\ \frac{a^{3}}{\overrightarrow{a^{4}}}\end{array}\right)=A^{-1} B$.

Since this system is taken from practical applications without exact solution, then we test the accuracy of the solution presented by the present method, using test by $L_{2}$-norm:

For differential equation

$$
\begin{aligned}
\left\|\dot{x}_{1}-A_{11} x_{1}-A_{12} x_{2}-B_{1} u-f_{1}\right\|_{2} & \\
= & {\left[\int _ { 0 } ^ { 1 } \left[\left|\dot{x}_{11}-x_{13}+2 t+\frac{x_{12}+x_{13}+1}{2}-t\right|^{2}+\mid \dot{x}_{12}-x_{12}-x_{13}-2 x_{14}+\right.\right.} \\
& 2 t+\frac{x_{12}+x_{13}+1}{2}+\left.e^{-t}\right|^{2}+\mid \dot{x}_{13}-x_{11}-x_{12}-x_{13}+2 t+\frac{x_{12}+x_{13}+1}{2}- \\
& \left.\left.\left.\quad e^{-t}\right|^{2}+\left|\dot{x}_{14}-x_{12}+2 t+\frac{x_{12}+x_{13}+1}{2}-t-1\right|^{2}\right] d t\right]^{1 / 2} \\
= & 2 \times 10^{-5}
\end{aligned}
$$

And for equality constraint

$$
\left\|A_{21} x_{1}+f_{2}\right\|_{2}=\left[\int_{0}^{1}\left|x_{11}+x_{14}+t^{2}+t\right|^{2} d t\right]^{1 / 2}=4 \times 10^{-4}
$$

Which states the accuracy of the present approach even if the exact solution is unknown for the system.

Figure 5.3 shows the differential equality states for open loop control $u(t) \in \Delta_{u}$, where $\Delta_{u}$ is the class of admissible control. 


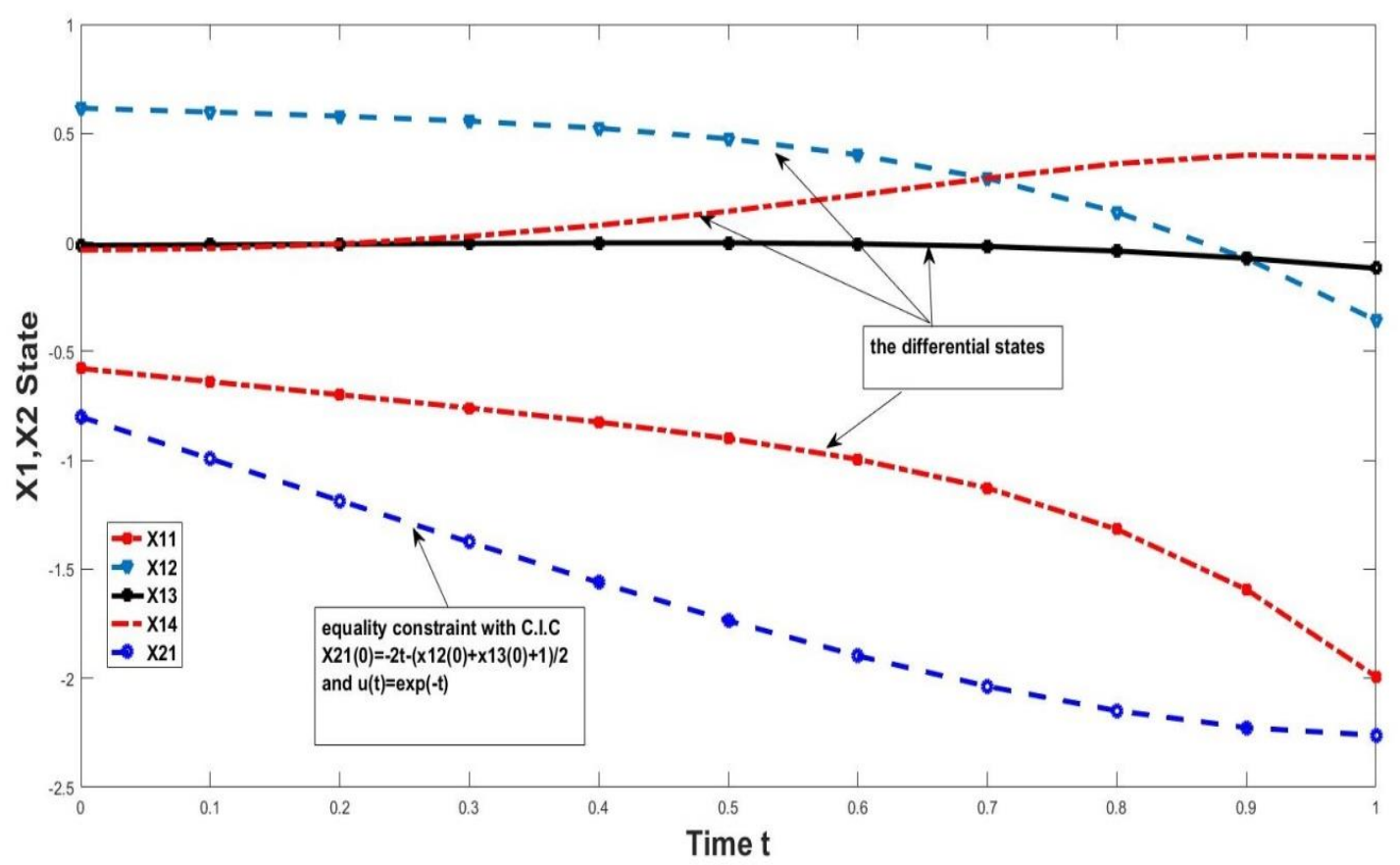

Figure 5.3-Differential-equality states for linear mechanical system of index two control model at $u(t)=e^{-t}$.

\section{Results and discussion}

The illustrated examples 5.1-5.3 are ranked from simple to more complex. The examples 5.1-5.2 are of semi explicit index-2, time-varying differential algebraic system, with known exact solutions. While the last example 5.3 is a descriptor system which firstly needs transformation, using singular value decomposition, to semi explicit DAEs, and needs to be taken from real life application without knowing its exact form solution.

These examples are taken as a test for the proposed method. By step by step implementation, the approximate solution is parameterized via polynomial base function, which is dense in $c\left[I, R^{n_{0}}\right]$. Even with reasonable small number of these polynomials with unknown coefficient, the obtained solutions are shown to be very accurate and efficient. Figures 5.1-5.3 show the excellent matching between the approximate solution, using the present method, and the given exact solution. The overall error value, using $L_{2}$ norm of the linear operators, showed very good results on $t \in\left[t_{0}, t_{f}\right], t_{f}>t_{0}$, for each example. The pointwise error in tables 5.1-5.4 demonstrated the good accuracy.

For example 5.3, due to the absence of the exact form solution, the $L_{2}$ norm errors for all the constraints (the differential and the algebraic) are adapted $\left(\left\|E_{1}\right\|_{2}=2 \times 10^{-5},\left\|E_{2}\right\|_{2}=4 \times\right.$ $\left.10^{-4}, E_{1} \triangleq L_{1} x-G_{1}(x), E_{2} \triangleq L_{2} x-G_{2}(x)\right)$ to test the convergence of the solution.

As an overall evaluation, the method has very good accuracy, being simple and effective as a tool to solve index 2, time-varying control DAEs.

\section{Conclusions}

As one can see, the present method is suitably applicable for an efficient class of index two DAEs with input $u(t)$ or even with semi-explicit index two, descriptor system. The method is easily implemented and a very good accuracy has been obtained, even for simple types of polynomial bases functions. This approach is reliable and efficient for this class of functions and can be extended to higher index DAEs (index greater than two).

\section{Acknowledgments}

The authors would like to thank Mustansiriyah University (www.uomustansiriyah.edu.iq), Baghdad, Iraq, for its support in the present work. 


\section{Reference}

[1] F.Soltanin, S.M.Karbassi, M.Dehghan,"Solution of the differential algebraic equations via homotopy perturbation method and their engineering applications", Inernational Journal of Computer Mathematics, vol.87, no.9, pp.1950-1974, 2019.

[2] F.Shakeri and M.dehghan, "Solution of a model describing biological species living together using the variational iteration method", Math. Comute. Modelling, vol. 48, pp.685-699, 2009.

[3] F.Soltanin, S.M.Karbassi, M.M.Hosseini,"Application of He's variational iteration method for solution of differential-algebric equations", Chaos,Solitons Fractals, vol. 41, no. 1, pp.436-445, 2009.

[4] E. Griepentroy and P. Marz, Differential-algebraic equations and their numerical treatment, Teubner Verlag, Liepizig, Germann, 1986.

[5] H. Mathew and V. John, "The Implicit Function Theorem with Applications in Dynamics and control," 48th AIAA Aerospace Science Meeting Including the new Horizons Forum and Aerospace Exposition, 4-7 January, Orland, Florida, 2010.

[6] L.Jay, "Specialized Runge-Kutta methods for index 2 differential-algebric equations", Math Comput, vol. 75, pp. 641-654, 2005.

[7] K.F.Brenan, S.L.Campbell and L.R.Petzold, Numerical Solution of Initial-Value Problems in Differential Algebraic Equations, Elsevier, NewYork, 1989.

[8] M.M.Hosseini, "Adomain decomposition method for solution of differential algebraic equations", J. Comput Appl.Math. vol. 197, pp.495-501, 2006.

[9] R. März, "Higher-index differential-algebraic equations: analysis and numerical treatment," Numerical Analysis, vol. 24, pp. 199-222, 1990.

[10]S. Karimi Vanani and A. Aminataei, "Numerical solution of differential algebraic equations using a multiquadric approximation scheme," Mathematical and Computer Modelling, vol. 53, no. 5-6, pp. 659-666, 2011.

[11]Radhi A. Zaboon, G. F. Abd,"Approximation solution of a linear descriptor dynamic control system via a non-classical variational approach ", IOSR Journal of Math. , vol.11, no.1, pp.1-11, 2015.

[12]Radhi A. Zaboon, G. F. Abd,"Solvability of Time-Varying Descriptor Systems Using NonClassical Variational Approach ", Mathematical Theory and Modeling, vol.5, no. 12, pp.74-82, 2015.

[13]G. F. Abd, Radhi A. Zaboon, "Solvability and Qualitative Properties of Non-linear Descriptor Systems," Lambert Academic Publishing, ISBN:978-659-86339-4, 2017.

[14]G. R. Duan, Analysis and design of Descriptor Linear System, Advances in Mechanics and Mathematics, Springer Science and Business Media, Springer, 2010.

[15]C. Ercan and B. Mustafa, "Arbitrary order numerical method for solving differential-algebraic equation by Pade series," Applied Mathematics and Computation, vol. 137, pp. 57-65, 2003.

[16] Y. Z. Sun, "Solvability of higher index time-varying linear differential-algebraic equations," Acta Mathematica Scientia, vol. B, no. 1, pp. 77-92, 2001.

[17]S. G. Krantz, Harold R. Parks, The Implicit Function Theorem History, Theory, and Application, Birkhauser, Berlin, second addition, 2002.

[18]U. M. Ascher, L. R. Petzold, Projected Implicit Runge-Kutta methods for Differential-Algebric Equations", SIAM J. Numerical Analysis, vol. 28, pp. 1097-1120, 1990.

[19] F. Magri, , "Variational Formulation for every Linear Equation", Int.J.Engng.Sci.,vol.12, pp:537-549,1974. 